\title{
Biosynthèse des protéines de réserve et formation des corps protéiques dans la graine de lupin jaune (Lupinus luteus L, Légumineuses)
}

\author{
MA Esnault, A Merceur, J Citharel \\ Université de Rennes l, laboratoire de physiologie végétale, campus de Beaulieu, F 35042 Rennes Cedex, France
}

(Reçu le 18 septembre 1992; accepté le 15 février 1993)

\begin{abstract}
Résumé - Le dépôt des protéines et la formation des corps protéiques dans la graine du lupin jaune sont observés en microscopie optique à différents stades de la séminogenèse. Parallèlement, la biosynthèse des protéines de réserve est suivie par l'étude de la composition en polypeptides des protéines totales et de fractions purifiées au moyen de l'électrophorèse et de l'utilisation de 2 sérums spécifiques des conglutines $\gamma$ et $\alpha$. La conglutine $\gamma$ est synthétisée (22 $\mathrm{j}$ après anthèse [JAA]) avant le dépôt de protéines dans les vacuoles. Durant la période de forte activité synthétique de la graine (27-33 JAA), les conglutines $\alpha$ (légumine) et $\delta$ (fraction 2S) apparaissent sous leurs formes matures. Les 2 types de corps protéiques, l'un à matrice homogène et l'autre hétérogène, sont déjà visibles. Le sérum anticonglutine $\gamma$ ne permet pas la détection d'un précurseur de la conglutine $\gamma$. Le sérum anticonglutine $\alpha$ met en évidence la présence de polypeptides de 84,74 et $62 \mathrm{kDa}$ au stade $27 \mathrm{JAA}$, suggérant que le processus de synthèse de la conglutine $\alpha$ est le même chez le lupin jaune que chez d'autres lupins.
\end{abstract}

globuline / corps protéique / maturation / légumineuse / immunologie / microscopie photonique

Summary - Biosynthesis of storage proteins and protein body formation in the seeds of the yellow lupin (Lupinus luteus $\mathrm{L}$, Leguminosae). Protein deposit and protein body formation in the seed of the yellow lupin were observed by optical microscopy for different stages of maturation. At the same time, storage protein biosynthesis was assessed by study of polypeptide composition of whole proteins and purified fractions, through electrophoresis and by the use of 2 specific sera. Conglutin $\gamma$ was synthesized from 22 DAA (day after anthesis) prior to protein deposition in the vacuoles. During the period of intense synthetic activity in the seed (27-33DAA), mature forms of conglutins $\alpha$ (legumin) and $\delta$ (2S fraction) appeared. Two types of protein bodies were detected depending on whether the matrices were homogeneous or heterogeneous. No conglutin $\gamma$ precursor could be detected by serum anti-conglutin $\gamma$. Polypeptides of 84,74 and $62 \mathrm{kDa}$ present at 27 DAA were recognized by serum anti-conglutin $\alpha$, suggesting that the biosynthetic process of conglutin $\alpha$ was the same in yellow lupin as in other lupins.

globulin / protein body / maturation / Leguminosae / immunology / photonic microscopy

\section{INTRODUCTION}

Les protéines de réserve des graines des lupins sont constituées de globulines (87 à $90 \%$ des protéines totales) et d'albumines (10-13\%). Chez le lupin jaune (Lupinus luteus L), 5 globulines ont été caractérisées (Esnault et al, 1991) : les conglutines $\beta 1$ et $\beta 2$ (vicilines), les conglutines $\alpha$ (légumine), $\gamma$ et $\delta$. Ces 2 dernières sont spécifiques des lupins.
La conglutine $\gamma$ peut être rapprochée d'une fraction $10 \mathrm{~S}$ du soja décrite par Sato et al (1987). Elle est formée chez les lupins par l'association de monomères constitués de 2 polypeptides liés par des ponts disulfures, de taille variable suivant les espèces. Chez le lupin jaune, le monomère de $41 \mathrm{kDa}$ contient 2 polypeptides de 33 et $17 \mathrm{kDa}$ (Esnault et al, 1991). La conglutine $\delta$ du lupin jaune est la fraction $2 S$ identifiée par Joubert (1955). Chez $L$ augustifo-

Abréviations : GM : graines mûres; GAR : immunoconjugué de chèvre antilapin; JAA : jours après l'anthèse; ME : 2mercaptoéthanol; SDS : dodécyl sulfate de sodium; Tris : trishydroxyméthyl aminométhane. 
lius, elle est présente sous 2 formes. La première (conglutine $\delta 2$ ) est composée de 2 sousunités de 9 et $4,5 \mathrm{kDa}$ associées par des ponts disulfures (Lilley et Inglis, 1986). La seconde (conglutine $\delta 1$ ) serait un dimère de la première (Lilley, 1986). Restani et al (1981) décrivent chez $L$ albus, une fraction $9 b$, la conglutine $\delta$, composée de 2 sous-unités, l'une de 21,6 kDa formée par l'association de 2 polypeptides de $10,5 \mathrm{kDa}$ et l'autre de $13,7 \mathrm{kDa}$ contenant au moins un polypeptide de $12,8 \mathrm{kDa}$. Dans le lupin jaune, la conglutine $\delta$ serait constituée des 2 types de protéines (Esnault et al, 1992). Une forme correspondant à la conglutine $\delta 2$ a pu être purifiée chez le lupin jaune (résultats non publiés).

Les conglutines des lupins sont synthétisées sous la forme de polypeptides précurseurs, de masses moléculaires supérieures à celle des polypeptides de la protéine mature. Le processus de maturation comporte d'abord l'élimination de la séquence signal, mise en évidence chez le lupin à feuilles étroites (Gayler et al, 1984) et le lupin jaune (Deckert et Gwozdz, 1991) pour les conglutines $\alpha, \beta$ et $\gamma$. Les précurseurs subissent ensuite des modifications post-traductionnelles semblables à celles décrites pour les protéines de réserve des légumineuses (Johnson et al, 1985). Ainsi, le précurseur de la conglutine $\gamma$ de $51 \mathrm{kDa}$ chez $L$ angustifolius (Johnson et al, 1985 ) et $L$ luteus (Deckert et Gwozdz, 1991) et de $45 \mathrm{kDa}$ chez $L$ albus (Citharel et Delamarre, 1989) est graduellement modifié pour aboutir aux polypeptides présents dans la graine mûre. Le précurseur de la conglutine $\delta 2$ de $L$ angustifolius subit des modifications post-traductionnelles similaires se traduisant par la perte d'un peptide signal de 22 puis d'un fragment de 13 acides aminés, libérant ainsi les 2 polypeptides de la protéine (Gayler et al, 1990).

Les protéines de réserve sont déposées dans des organites cellulaires spécialisés, les corps protéiques, dans lesquels sont effectuées les dernières étapes de leur maturation (Johnson et al, 1985). Chez les Légumineuses, les corps protéiques contiennent des globoïdes cristallins inclus dans une matrice de type protéique (Lott et Buttrose, 1978; Lott, 1981). Chez certaines Papilionacées, ils se distinguent par une dualité très marquée au niveau de la structure et de la localisation dans le cotylédon : les uns, à matrice homogène, sont présents sur la face adaxiale; les autres, avec une matrice hétérogène contenant de nombreuses alvéoles et des globoïdes cristal- lins, sont situés sur la face abaxiale. Ils ont été décrits chez le lupin jaune (Sobolev et al, 1976; Mlodzianowski, 1978), chez Cytisus scoparius (Citharel et Citharel, 1985) et dans une grande majorité d'espèces de la tribu des Génistées (Citharel et Citharel, 1986). La formation des corps protéiques a été étudiée chez le lupin jaune par Wozny et al (1984), et chez le lupin blanc par Davey et Van Staden (1978) et Le Gal et Rey (1986).

Les mécanismes de synthèse des protéines de réserve et leur dépôt dans les corps protéiques au cours de la maturation de la graine des Légumineuses sont connus chez certaines espèces (pois, soja, fèverole...). Toutefois, si le schéma général semble commun, des spécificités peuvent exister chez certaines espèces, ou pour des protéines particulières. Pour le lupin jaune, ces connaissances demeurent fragmentaires et incomplètes. Comparé aux autres protéagineux, celui-ci présente le double intérêt de posséder des graines parmi les plus riches en protéines $(44 \%)$ et de très bien se développer sur les sols pauvres et sableux, difficiles à exploiter. Ce travail a donc pour but de compléter l'étude des protéines de réserve du lupin jaune, et en particulier, celle portant sur leur biosynthèse, et de leur dépôt dans les corps protéiques.

\section{MATÉRIEL ET MÉTHODES}

L'étude concerne les graines de lupin jaune de la variété Sulfa, sélectionnées par la station d'amélioration des plantes fourragères (INRA) de Lusignan pour leur basse teneur en alcaloïdes.

\section{Extraction des globulines totales aux différents stades de maturation}

Les graines immatures (tableau I) sont congelées dans l'azote liquide puis conservées à $-20^{\circ} \mathrm{C}$. Elles sont broyées dans un mortier, dans l'azote liquide. Les protéines sont extraites en agitant le broyat dans un tampon Tris- $\mathrm{HCl} 50$ mmol. $\mathrm{I}^{-1}, \mathrm{pH} 8,3$ contenant du $\mathrm{NaCl} 1,5$ mol. $\mathrm{I}^{-1}$ à $4{ }^{\circ} \mathrm{C}$, pendant $2 \mathrm{~h}(1 / 10 ; \mathrm{p} / \mathrm{v})$. L'extrait est mélangé avec de l'acide trichloro-acétique à $20 \%(\mathrm{v} / \mathrm{v})$. Après un repos de $2 \mathrm{~h}$ à $4^{\circ} \mathrm{C}$, les protéines précipitées sont isolées par une centrifugation de 20 min à $17000 \mathrm{~g}$ et $4{ }^{\circ} \mathrm{C}$, puis lavées 3 fois avec un volume du mélange éther-alcool $(1 / 1 ; v / v)$ identique au volume du tampon d'extraction. Les protéines sont ensuite solubilisées dans un tampon Tris- $\mathrm{HCl} 0,5$ mol. I $^{-1}$ $\mathrm{pH}$ 6,8 pour l'analyse électrophorétique. 


\section{Purification des globulines}

Les différentes fractions de globulines sont extraites à partir de graines immatures (stade 6) ou mûres, puis fractionnées par chromatographie d'échange d'ions et de filtration sur gel selon le protocole décrit précédemment par Esnault et al (1991). Le monomère de $41 \mathrm{kDa}$ de la conglutine $\gamma$, est isolé par électrophorèse préparative sur gel contenant $10 \%$ d'acrylamide et $2 \%$ de SDS $(150 \times 140 \times 1,5 \mathrm{~mm})$ selon la technique de Laemmli (1970). Les dépôts protéiques sont de $500 \mu \mathrm{g}$ par puits. La migration est réalisée sous un courant constant de $30 \mathrm{~mA}$ par gel (tension de 150 volt), dans un tampon Tris 25 mmol..$^{-1}$-glycine $192 \mathrm{mmol}^{-1}$, de $\mathrm{pH} 8,6$ pendant $4-5 \mathrm{~h}$. A la fin de la migration, le polypeptide est localisé sur le gel par une coloration rapide des 2 pistes adjacentes, au bleu de Coomassie R250. Il est désorbé en laissant la portion centrale du gel le contenant en contact avec un tampon Tris- $\mathrm{HCl} 0,5$ mmol. $1^{-1}, \mathrm{pH} 6,8$, à $4{ }^{\circ} \mathrm{C}$, pendant une nuit. L'extrait, après centrifugation, est alors dialysé contre de l'eau distillée, à $4{ }^{\circ} \mathrm{C}$, pendant $24 \mathrm{~h}$ puis lyophilisé. La pureté de la fraction est contrôlée après chaque isolement par électrophorèse monodimensionnelle.

\section{Electrophorèses monodimensionnelles}

Les extraits protéiques sont analysés sur des gels $(150 \times 120 \times 1 \mathrm{~mm})$ d'acrylamide soit en gradient de $5-13 \%$ sans SDS pour les protéines natives, soit à concentration constante $(12,5 \%)$ et $2 \%$ en SDS pour les protéines dénaturées. Les dépôts sont d'environ $40 \mu \mathrm{g}$ par puits. Les électrophorèses sont réalisées sous une intensité constante de $30 \mathrm{~mA}$ par gel. Les polypeptides sont révélés au bleu de Coomassie R250. Chaque gel est étalonné par des protéines de référence : lysozyme (14,4 kDa), inhibiteur de trypsine de soja $(21,5 \mathrm{kDa})$, anhydrase carbonique (31 kDa), albumine d'œuf $(42,7 \mathrm{kDa})$, albumine de sérum de bœuf $(66,2 \mathrm{kDa})$ et phosphorylase $\mathrm{B}(97,4 \mathrm{kDa})$.

\section{Préparation des sérums et technique d'immuno-empreinte sur membrane de nitrocellulose}

Des sérums sont obtenus à partir du monomère de $41 \mathrm{kDa}$ de la conglutine $\gamma$ et la conglutine $\alpha$ purifiée (Esnault et al, 1991). Quatre injections sous-cutanées de $1 \mathrm{mg}$ de protéines dans $0,5 \mathrm{ml}$ d'adjuvant de Freund (complet à la première injection puis incomplet) et $0,5 \mathrm{ml}$ d'eau sont effectuées sur des lapins, à raison d'une par semaine. La spécificité des sérums est vérifiée par la technique d'immuno-empreinte. Les protéines, séparées par électrophorèse en gel d'acrylamide à $12,5 \%$ et en présence de SDS, sont transférées pendant 30 min sur une membrane de nitrocellulose (nitroplus, Bioblock) dans une cuve Semiphor TE70 (Hoefer Scientific Instruments). Le tampon Tris (25 mmol. $\left.\left.\right|^{-1}\right)$-glycine(192 mmol. $\mathrm{I}^{-1}$ ), pH 8,6 contient du méthanol à $20 \%(\mathrm{v} / \mathrm{v})$ à l'anode et du SDS à $0,1 \%(v / v)$ à la cathode. La révélation est effectuée avec les sérums dilués au $1 / 1000^{\mathrm{e}}$ dans du tampon phosphate $\mathrm{pH} 7,5\left(\mathrm{Na}_{2} \mathrm{HPO}_{4} 8 \mathrm{mmol} . \mathrm{I}^{-1}, \mathrm{KH}_{2} \mathrm{PO}_{4} 1,5\right.$ mmol. $\left.\mathrm{I}^{-1}, \mathrm{NaCl} 137 \mathrm{mmol}^{-1}, \mathrm{KCl} 2,7 \mathrm{mmol}^{-1}\right)$ et un immunoconjugué, le GAR (GAR/lgG(H+L)/PO, TEBU) portant une peroxydase. La conglutine $\alpha$ est visualisée par incubation de la membrane dans un mélange contenant $50 \mu \mathrm{l} \quad \mathrm{d}^{\prime} \mathrm{H}_{2} \mathrm{O}_{2}$ à $30 \%, 75 \mathrm{ml}$ du tampon phosphate et $15 \mathrm{ml}$ de chloronaphtol à $3 \%$ dans le méthanol, la conglutine $\gamma$ à l'aide du luminol selon le protocole préconisé par le fabricant (Amersham). L'absence de réaction des 2 sérums avec les autres globulines du lupin jaune a été vérifiée dans ces conditions.

\section{Préparations microscopiques}

Des fragments de cotylédons de $1 \mathrm{~mm}^{3}$ sont prélevés à partir de graines immatures (tableau I). Ils sont fixés dans une solution de glutaraldéhyde à $2,5 \%$ dans un

Tableau I. Caractérisation des différents stades de maturation de la graine de lupin jaune.

\begin{tabular}{|c|c|c|c|c|c|c|c|c|}
\hline & 1 & 2 & 3 & $\begin{array}{l}\text { Stades } \\
4\end{array}$ & 5 & 6 & 7 & $\begin{array}{l}\text { Graines } \\
\text { mûres }\end{array}$ \\
\hline $\begin{array}{l}\text { JAA } \\
\text { Graine }(m g)^{\star}\end{array}$ & $\begin{array}{l}14 \\
<60\end{array}$ & $\begin{array}{l}17 \\
70-90\end{array}$ & $\begin{array}{l}20 \\
100-140\end{array}$ & $\begin{array}{l}22 \\
150-160\end{array}$ & $\begin{array}{l}27 \\
200-220\end{array}$ & $\begin{array}{l}31 \\
270-310\end{array}$ & $\begin{array}{l}33 \\
320-350\end{array}$ & $\begin{array}{l}70 \\
140 \pm 20\end{array}$ \\
\hline $\begin{array}{l}\text { Masse d'un } \\
\text { cotylédon (mg) }\end{array}$ & non formé & 7 & 12 & 24 & 54 & 84 & 86 & 54 \\
\hline
\end{tabular}

\footnotetext{
* Les valeurs des masses de matières fraîches sont établies sur 100 graines pour chaque stade. * La masse moyenne d'un cotylé-
} don est établie sur 20 cotylédons. 
tampon caccodylate de sodium-HCl 0,2 mmol.t-1 $(\mathrm{pH}$ $7,2)$, pendant $2 \mathrm{~h}$ à $4^{\circ} \mathrm{C}$. Après rinçage dans le même tampon mais $0,1 \mathrm{~mol} . \mathrm{I}^{-1}$, ils sont incubés dans le tétroxyde d'osmium à $1 \%$ dans le tampon caccodylate $0,2 \mathrm{mmol} . \mathrm{I}^{-1} \mathrm{pH} 7,2(1 / 1 ; \mathrm{v} / \mathrm{v})$, à $4{ }^{\circ} \mathrm{C}$, pendant $2 \mathrm{~h}$ à l'obscurité. Les échantillons sont ensuite déshydratés dans des bains successifs d'alcool à $70^{\circ}, 90^{\circ}, 100^{\circ}$ puis d'oxyde de propylène. Ils sont alors inclus dans de l'Epon (résine époxy). Les coupes semi-fines de 1 à $2 \mu \mathrm{m}$ sont réalisées par un microtome Reichert puis colorées au bleu de toluidine à $1 \%$. Les observations et photographies sont effectuées en microcopie photonique.
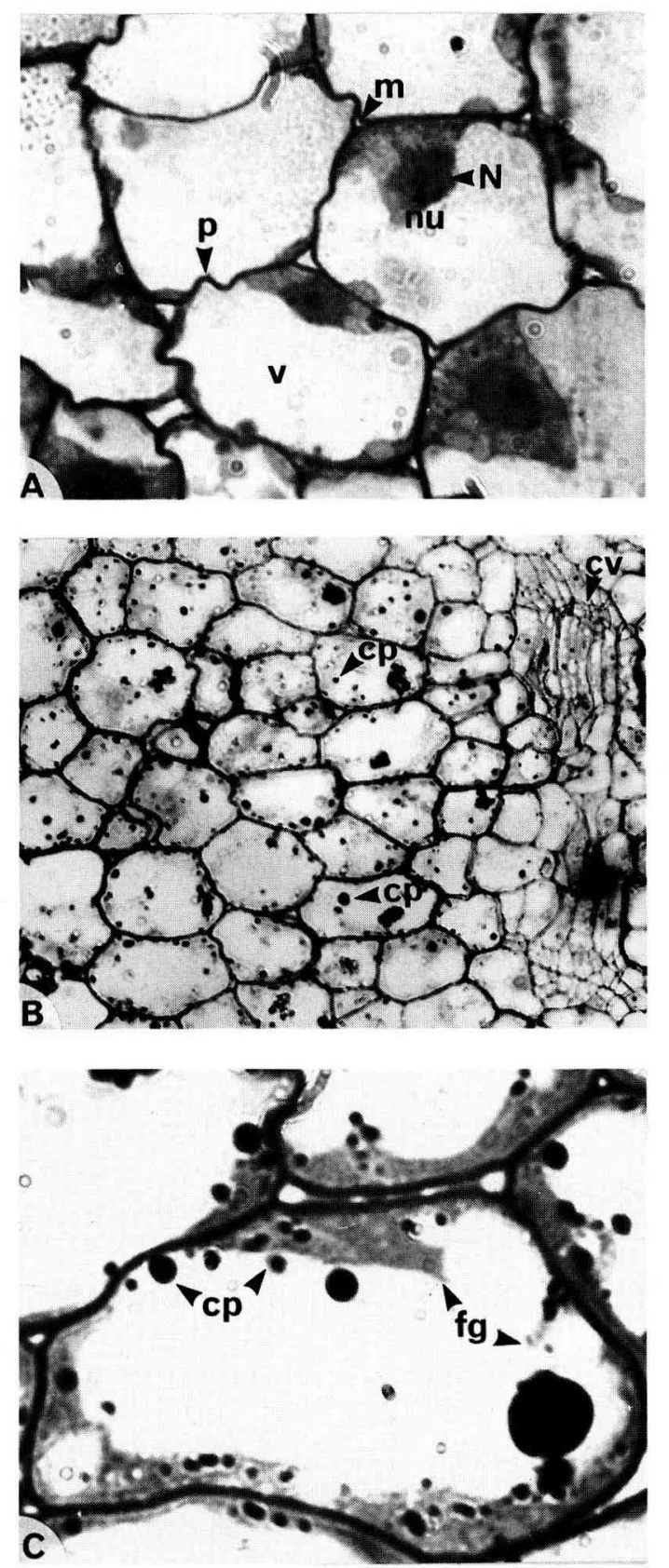

\section{RÉSULTATS}

\section{Caractérisation des stades de maturation}

Les divers stades immatures sont caractérisés par la durée du développement de la graine (JAA) et les masses de matière fraîche de la graine et des cotylédons (tableau I). Ces bases nous ont permis d'obtenir des résultats reproductibles pour 3 cycles de végétation.
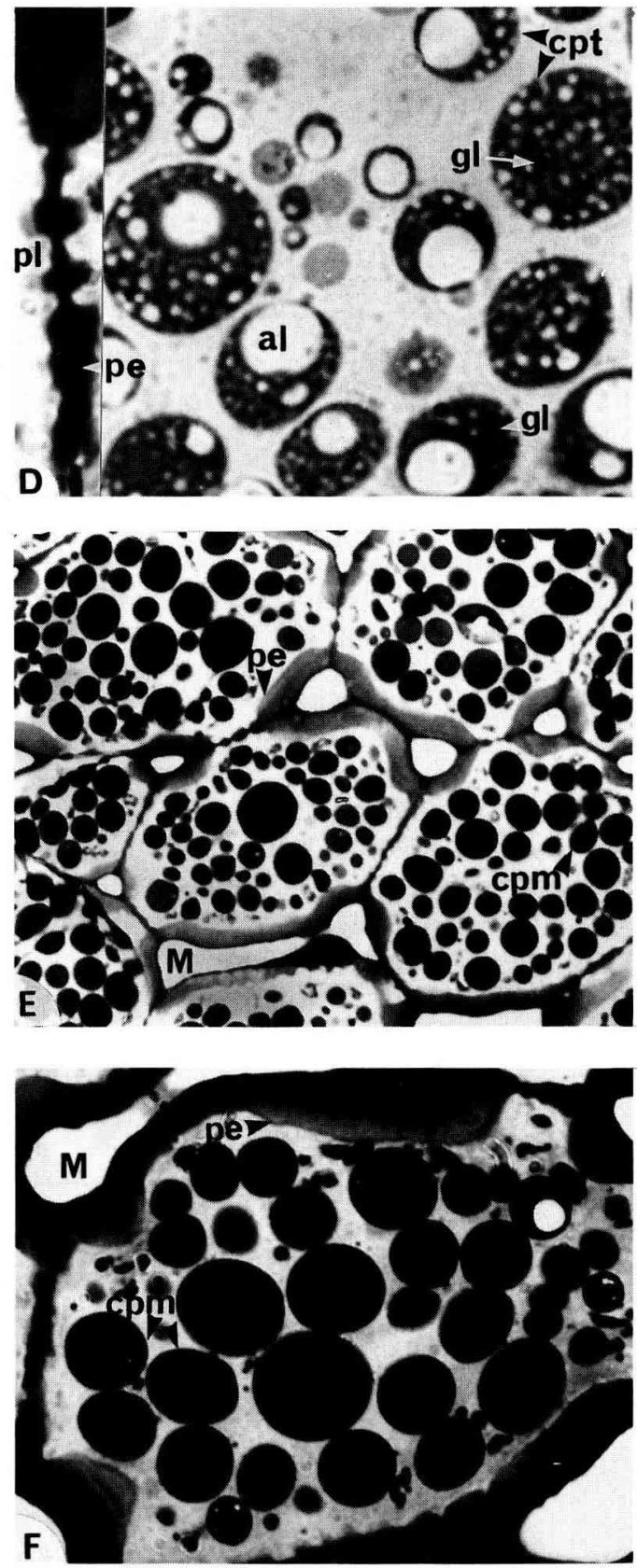

Fig 1. Coupes semi-fines de cotylédons de $L$ luteus, à différents stades de la séminogenèse. A. Stade $4(x 1200)$, $m:$ méat réduit, $N$ : noyau, nu : nucléole, $p$ : paroi cellulaire, mince et sineuse, $v:$ vacuole. B, C. Stade $5, B(x 300)$ et $C$ ( $\times 1200)$, cp: corps protéique de taille variable, sur le pourtour de la vacuole, cv : faisceau criblo-vasculaire, fg : fragmentaire de la vacuole. $D$. Stade 6 , vues de la membrane et des corps protéiques $(x 1200)$, al : alvéole, cpt : corps protéiques hétérogènes, $g l$ : globoïdes cristallins, pe : épaississement de la paroi interne, pl : emplacement des plasmodesmes. E, F. Stade 7, E (x 400) et $F(x 1200)$, cpm : corps protéiques homogènes, $M$ : méat important, pe : épaississement de la paroi interne. 
Dépôt des protéines et formation des corps protéiques

Aux stades 2 et 4 de l'ontogenèse, les cellules des parenchymes des faces adaxiale et abaxiale des cotylédons présentent :

- des parois fines avec un contour très sineux;

- une grande vacuole centrale, entourée par un cytoplasme réduit occupant la majeure partie du volume cellulaire;

- un noyau lobé, placé latéralement avec un nucléole bien visible et quelques chromocentres périphériques (fig 1A). Aucun dépôt protéique n'est décelable.

Au stade 5 , on observe une fragmentation du vacuome (fig $1 \mathrm{~B}, \mathrm{C}$ ). De petits dépôts protéiques fortement colorés au bleu de toluidine sont identifiables sur le pourtour des vacuoles. Le noyau et le nucléole sont encore bien visibles dans le cytoplasme périphérique. La paroi cellulaire est plus épaisse qu'aux stades précédents et les invaginations ont pratiquement disparu.

Aux stades 6 et 7 , d'importants changements interviennent (fig 1D, E, F). Les corps protéiques, bien formés, sont répartis en 2 zones avec des structures differentes. Ceux de la face adaxiale du cotylédon, lieu du futur parenchyme palissadique ont une matrice homogène. Ceux de la face abaxiale, lieu du futur parenchyme lacuneux, sont hétérogènes, ayant une matrice contenant des globoïdes cristallins et des "alvéoles" claires, de taille variable. En microscopie optique, les globoïdes cristallins présentent une réfringence rouge caractéristique. La paroi cellulaire est alors très épaisse surtout au niveau des espaces intercellulaires, méats et petites lacunes. Ailleurs, elle est moins épaisse et présente de nombreux plasmodesmes.

Dans la graine mûre, les corps protéiques occupent la totalité de la cellule.

\section{Biosynthèse des polypeptides au cours de la maturation de la graine}

Les graines les plus immatures (stades 1-5) contiennent un grand nombre de polypeptides (fig 2) avec des masses s'échelonnant entre 97 et $20 \mathrm{kDa}$. Ceux de $52,40,32$ et $20 \mathrm{kDa}$ prédominent. La présence de 2-mercaptoétharol ne modifie la masse d'aucun polypeptide (données non montrées). A partir du stade 6 , des changements notables apparaissent. Cinq nouveaux po- lypeptides de $85,76,65,50$ et $44 \mathrm{kDa}$ sont révélés. La bande de $20 \mathrm{kDa}$ visible dans les stades précédents a disparu alors que 2 autres de 18 et $13,5 \mathrm{kDa}$ apparaissent. Dans la graine mûre, celles-ci disparaissent au profit de 2 autres de 14,5 et $10 \mathrm{kDa}$, caractéristiques de la conglutine $\delta$ (Esnault et al, 1992) tandis que les bandes de 85,76 et $65 \mathrm{kDa}$ sont toujours mises en évidence. Celles de 50 et $44 \mathrm{kDa}$ ne sont plus décelées mais 2 nouvelles de 48 et $41 \mathrm{kDa}$ sont visibles en forte concentration.

La technique d'immuno-empreinte montre que la conglutine $\gamma$ mature est révélée faiblement à partir du stade 4 (22 JAA) mais surtout à partir du stade 5 (27 JAA). Dès ces stades, le monomère de $41 \mathrm{kDa}$, caractéristique de cette protéine, est visible (fig 3 ). II est scindé par le 2mercaptoéthanol en fragments de 33 et $17 \mathrm{kDa}$. Un polypeptide de $45 \mathrm{kDa}$ est aussi révélé dans ces conditions. Sa présence occasionnelle avec la conglutine $\gamma$ purifiée suggère qu'il proviendrait de la dissociation incomplète de la sous-unité de $41 \mathrm{kDa}$. Aucun précurseur ne peut être mis en évidence par la technique d'immuno-empreinte.

La conglutine $\alpha$ mature est représentée par des bandes de masses comprises entre 43 et 66 $\mathrm{kDa}$ (Esnault et al, 1991). Les bandes majeures de 62, 60, 51 et $43 \mathrm{kDa}$ sont bien reconnues par le sérum anti-conglutine $\alpha$ (fig 4). Ces sousunités sont décelées à partir du stade 5 . Une bande de $84 \mathrm{kDa}$ (a) n'est visible qu'au stade 5 . Un polypeptide de $62 \mathrm{kDa}$ (c) est reconnu par le

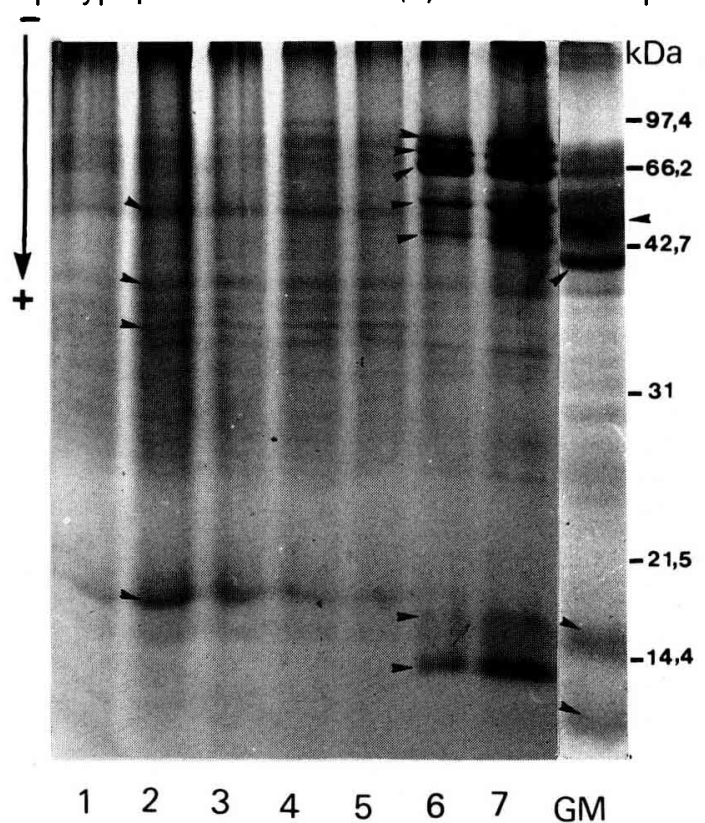

Fig 2. Electrophorèse en présence de SDS et en l'absence de ME, des protéines extraites à partir de graines immatures (stades $1-7$ ) et mûres (GM). Les polypeptides faisant l'objet d'un commentaire dans le texte sont signalés par des flèches. 


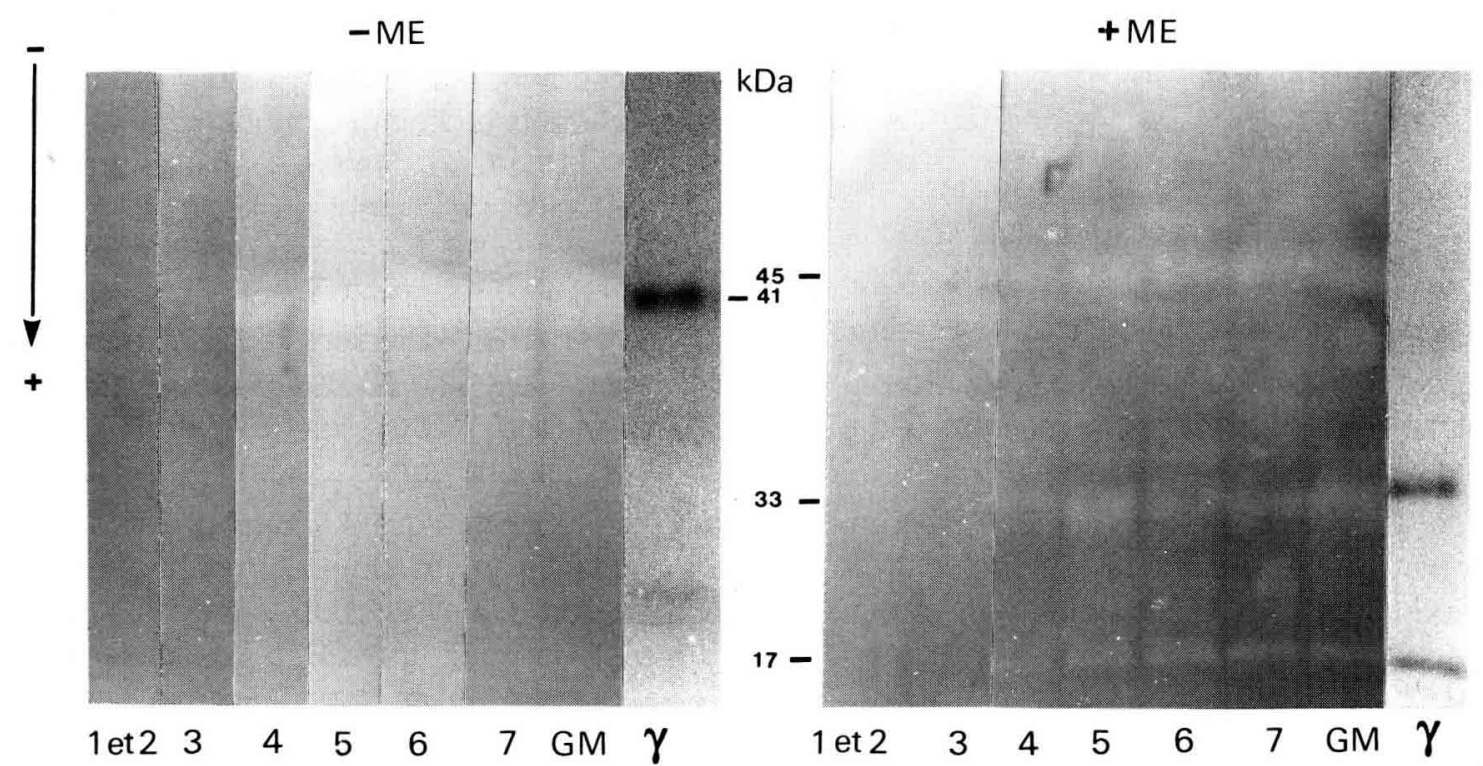

Fig 3. Révélation, après immuno-empreinte, des protéines extraites à partir de graines immatures et mûres, par le sérum anticonglutine $\gamma$ ( $\gamma$ : conglutine $\gamma$ révélée par le bleu de Coomassie).

sérum dès les premiers stades jusqu'à la graine mûre, un autre de $74 \mathrm{kDa}$ (b) à partir du stade 5 . lls pourraient correspondre à des sous-unités matures ou à des précurseurs de masse moléculaire très proche.

\section{Purification des protéines au stade 6 de la maturation}

Le stade 6 est caractérisé par une modification importante de la composition en sous-unités des globulines et de l'aspect des corps protéiques.

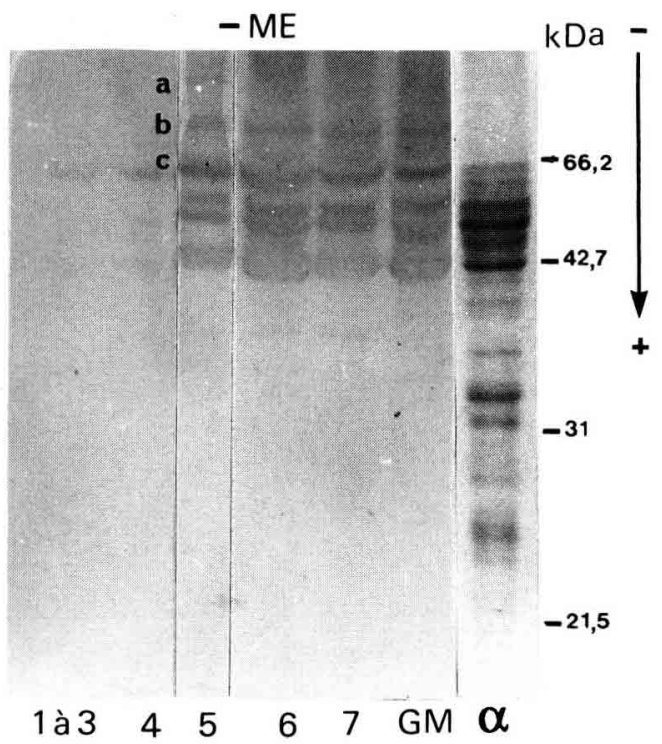

Les globulines présentes à ce stade ont donc été fractionnées par chromatographie d'échange d'ions selon un protocole identique à celui utilisé pour la graine mature (Esnault et al, 1991). Dans ces conditions, 4 fractions sont isolées : une non retenue $\left(F_{1}\right)$ et 3 fractions éluées à des concen-

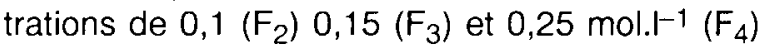
de $\mathrm{NaCl}$. Ces fractions ont été comparées aux fractions homologues isolées à partir de la graine mûre, par électrophorèses en absence ou en présence de SDS ou de ME.

La fraction $F_{1}$ donne par électrophorèse, en l'absence de SDS et de ME, une seule bande si-

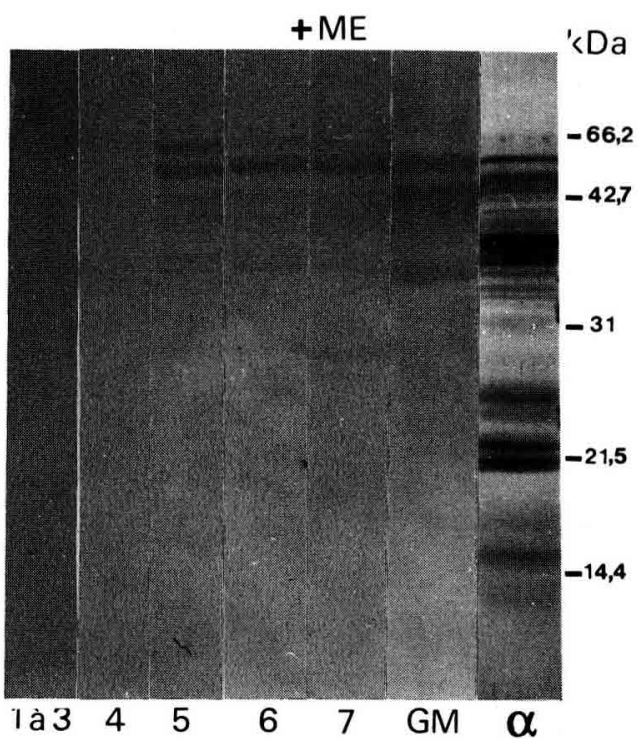

Fig 4. Révélation, après immuno-empreinte, des protéines extraites à partir de graines immatures et mûres, par le sérum anticonglutine $\alpha$ ( $\alpha$ : conglutine $\alpha$ révélée par le bleu de Coomassie). 
milaire à celle de la conglutine $\gamma$ (fig 5). En présence de SDS, elle est caractérisée comme la conglutine $\gamma$ par une bande majeure de $41 \mathrm{kDa}$ et des bandes mineures dont une de 43,5 kDa. La bande majeure est réduite par le 2mercaptoéthanol en 2 polypeptides de 33 et 17 kDa. Dans la fraction $F_{1}$, ce dernier, proportionnellement en plus faible concentration, est accompagné d'un polypeptide de $15 \mathrm{kDa}$.

La fraction $F_{2}$, pauvre en protéines, donne en l'absence de SDS une bande migrant à la même vitesse que la bande la plus mobile de la conglutine $\delta$ (fig 6 ). En présence de SDS, elle renferme 2 sous-unités de 14,5 et $10 \mathrm{kDa}$, réduites par le $\mathrm{ME}$ en trois polypeptides majeurs de $12 \mathrm{kDa}, 5$ $\mathrm{kDa}$ et $3,5 \mathrm{kDa}$, confirmant les similitudes avec la conglutine $\delta$.

La fraction $F_{3}$ est constituée, en l'absence de SDS, d'une seule bande ne correspondant à aucune des conglutines matures et, en présence de SDS, de sous-unités de masses comprises entre 88 et $45 \mathrm{kDa}$ et qui ne se scindent pas sous l'action du 2-mercaptoéthanol (fig 6). Elles sont différentes de celles de la conglutine $\beta$ (56 à $43 \mathrm{kDa}$ ) que l'on pourrait s'attendre à trouver dans cette fraction. Par ailleurs, les polypeptides de la conglutine $\delta$ y sont faiblement révélés.

Quelles que soient les conditions, les profils électrophorétiques de la fraction $\mathrm{F}_{4}$ sont similaires à ceux de la conglutine $\alpha$, montrant ainsi que celle-ci est déjà sous sa forme mature au

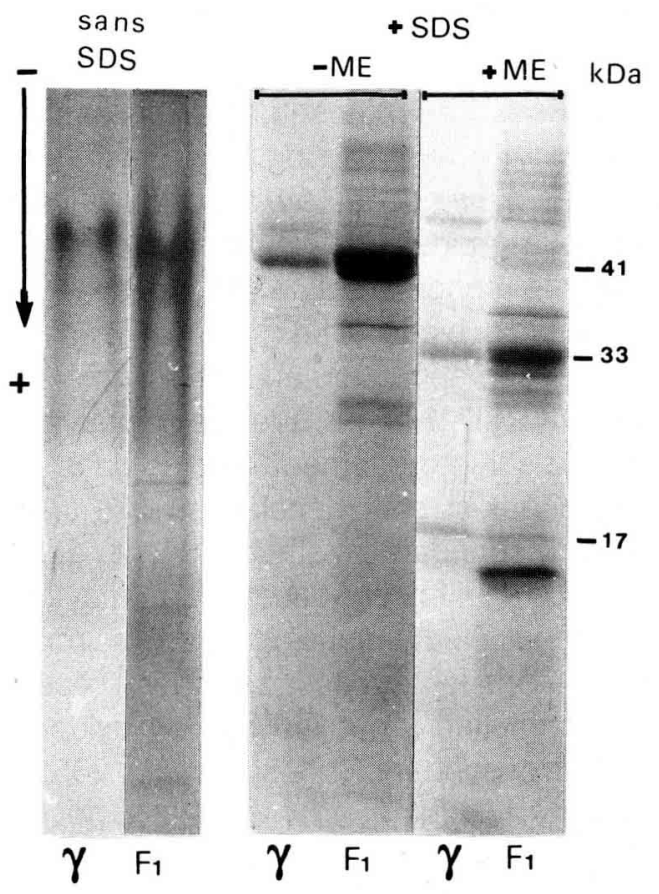

Fig 5. Électrophorèses de la conglutine $\gamma$ purifiée $(\gamma)$ et de la fraction chromatographique $F 1$ des globulines isolées au stade 6 , en l'absence ou en présence de SDS, en absence $(-)$ et en présence $(+)$ de ME.

stade 6 (fig 7). En présence de SDS, une différence majeure est toutefois à noter. Elle concerne l'existence de sous-unités de 88 à 70 $\mathrm{kDa}$, non modifiées par le 2-mercapto-éthanol et similaires à celles de la fraction $F_{3}$, suggérant une contamination.
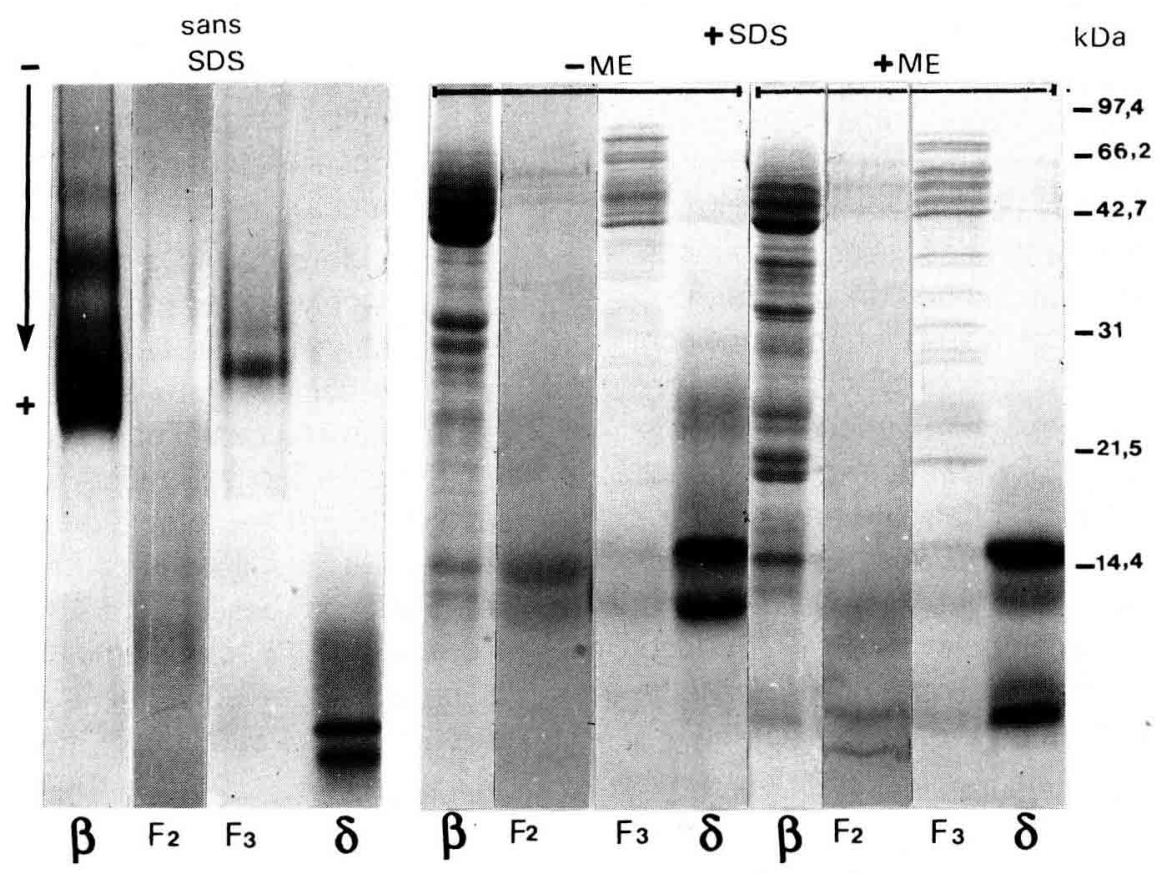

Fig 6. Électrophorèse des conglutines $\beta$ et $\delta$ purifiées $(\beta, \delta)$ et des fractions chromatographiques $F 2$ et $F 3$ isolées au stade 6 , en l'absence et en présence de SDS et de ME. 


\section{DISCUSSION}

L'étude de la biosynthèse des globulines des lupins a montré que la conglutine $\gamma$ est l'une des premières synthétisées au cours du développement de la graine. Citharel et Delamarre (1989) décèlent le monomère de $43 \mathrm{kDa}$ dès le $25^{\mathrm{e}} \mathrm{JAA}$ dans le lupin blanc. Chez le lupin jaune, nous montrons son apparition au 22 2 JAA sous forme mature $(41 \mathrm{kDa})$ avant que les dépôts de protéines ne soient nettement visibles dans les vacuoles. Ceux-ci sont décelés au $27^{e}$ JAA, période correspondant au stade II décrit par Wozny et al (1984) qui les ont observés au moyen de la microscopie électronique à transmission. Chez le lupin à feuilles étroites, seuls les précurseurs de la conglutine $\gamma$ ont été mis en évidence au stade correspondant (Johnson et al, 1985).

La période comprise entre le 27 et le $33^{3}$ JAA est cruciale pour la biosynthèse des globulines des lupins. Il a été montré, dans le lupin à feuilles étroites, qu'avant ce stade seuls les précurseurs des protéines sont marqués lors de synthèse in vitro ou in vivo. À partir du 33e JAA, les conglutines $\gamma$ et $\alpha$ achèvent leur maturation et les polypeptides apparaissent sous leur forme définitive (Duranti et al, 1991; Gayler et al, 1984; Blagrove et al, 1984; Johnson et al, 1985; Gayler et al, 1989). Dans le lupin jaune, le fractionnement des globulines présentes au 31 e JAA (stade 6) confirme l'existence de formes matures pour les conglutines $\alpha, \delta$ et $\gamma$. La structure quaternaire est donc déjà acquise à ce stade. L'électrophorèse en l'absence de SDS permet, en effet, d'obtenir des profils tout à fait semblables aux protéines matures. Toutefois, la conglutine $\delta$ ne semble pas organisée sous sa forme mature définitive, bien que ses polypeptides caractéristiques semblent déjà synthétisés. Les conglutines $\beta 1$ et $\beta 2$ achèvent leur maturation plus tardivement et n'ont pas encore acquis leur composition en sous-unités finales. En ce qui concerne l'organisation cellulaire pour cette période, les corps protéiques sont déjà bien formés et possèdent des globoïdes. C'est, en effet, une période d'accumulation intense de protéines (Gayler et al, 1984). On reconnaît déjà les 2 types de corps protéiques décrits par Sobolev et al (1976) avec leur localisation caractéristique. Cette période correspond au stade III de Wozny et al (1984).

Les sous-unités majeures de 45 à $88 \mathrm{kDa}$, présentent dans les fractions $F_{2}, F_{3}$ et $F_{4}$ pourraient être des précurseurs des vicilines et légumines. Decker et Gwozdz (1991) ont en effet dé-

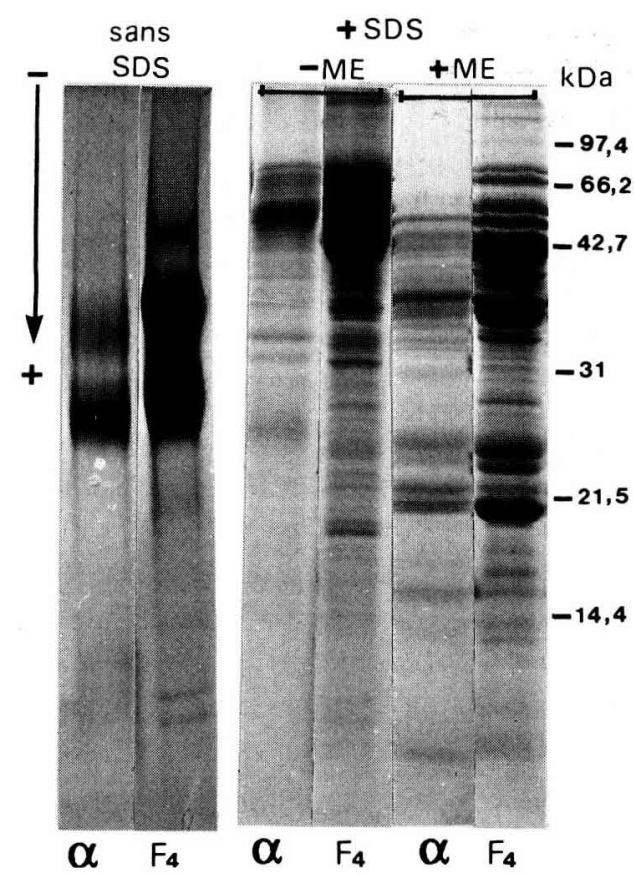

Fig 7. Electrophorèse de la conglutine $\alpha$ purifiée et de la fraction chromatographique $F_{4}$ isolée au stade 6 , en l'absence et en présence de SDS et de ME.

crit chez le lupin jaune des précurseurs de la conglutine $\beta$ compris entre 74 et $43 \mathrm{kDa}$. Le polypeptide de $84 \mathrm{kDa}$ reconnu par le sérum anticonglutine $\alpha$ au 27 e JAA pourrait être l'homologue du précurseur de $83 \mathrm{kDa}$ décelé chez le lupin à feuilles étroites par la même technique (Gayler et al, 1984). Ceux de 74 et $62 \mathrm{kDa}$, visibles essentiellement à ce stade, ont des masses très proches de celles des précurseurs de 72 et $64 \mathrm{kDa}$ décelés par Johnson et al (1985) chez $L$ angustifolius et de celles de 71 et 60 kDa observées par Deckert et Gwozdz (1991) chez L luteus.

La bande de $20 \mathrm{kDa}$ révélée aux stades 1-5 lors de l'analyse électrophorétique de l'ensemble des globulines, puis celles de 18 et $13,5 \mathrm{kDa}$ mises en évidence aux stades 6 et 7 présentent des ressemblances avec un précurseur décrit de la conglutine $\delta$ chez le lupin à feuilles étroites, à savoir une propréprotéine de 17765 Da perdant un peptide signal pour donner une préprotéine de $15538 \mathrm{Da}$ (Gayler et al, 1990). La perte d'une séquence de 13 acides aminés conduit ensuite à la formation des sous-unités de la protéine mature (9 558 et $4520 \mathrm{Da})$.

Johnson et al (1985) ont montré chez le lupin à feuilles étroites que la biosynthèse d'une protéine et ses modifications post-traductionnelles sont programmées pour être réalisées au cours 
du développement de la graine. Chez les lupins, le temps exceptionnellement long entre la période de biosynthèse des conglutines $\alpha$ et $\beta$ notamment et l'apparition de leurs formes matures serait dû à l'absence d'exo- ou endoprotéases nécessaires aux clivages des précurseurs. L'em- bryon ne synthétiserait ces enzymes que plus tardivement. Les périodes de maturation successives des précurseurs des différentes globulines conduiraient dont à émettre l'hypothèse de l'intervention de protéases différentes pour les 4 globulines majeures du lupin.

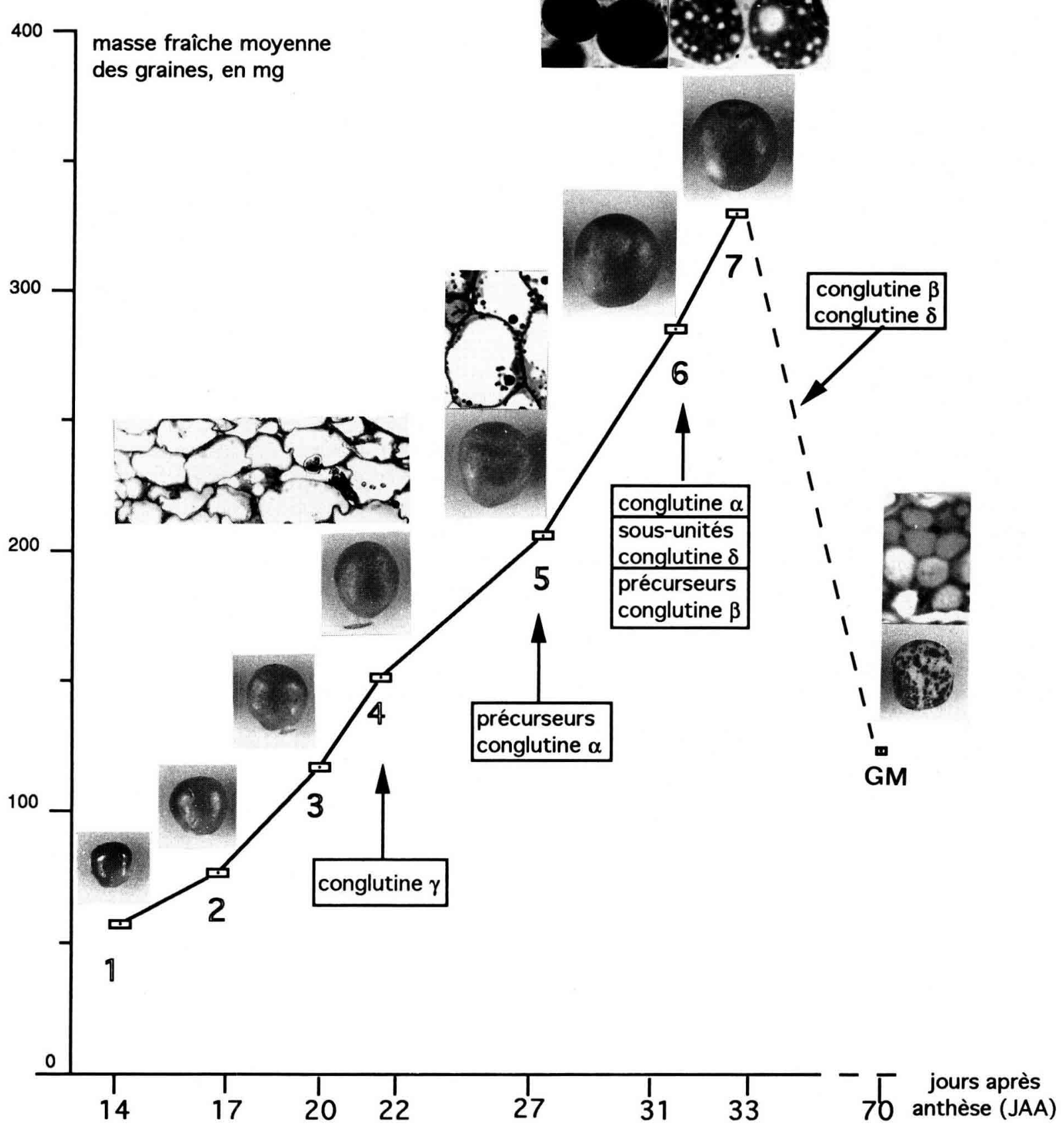

Fig 8. Aperçu global des différents événements intervenant au cours de la séminogenèse chez $L$ luteus en rapport avec la morphologie de la graine (grossissement $\times 1,5$ ), l'apparition des corps protéiques et les principales étapes de la biosynthèse des globulines. 


\section{RÉFÉRENCES}

Blagrove RJ, Lilley GG, Gayler KR, Johnson ED (1984) Biosynthesis and structure of lupin storage proteins. In: $3^{e}$ congrès int lupin. La Rochelle, 312324

Citharel L, Citharel $J(1985)$ Protein bodies from the cotyledons of Cytisus scoparius L (Link). Ultrastructure, isolation and subunit composition of albumin, legumin and vicilin. Planta 166, 39-45

Citharel L, Citharel J (1986) Dualité des corps protéiques du mésophylle adaxial et abaxial des cotylédons de quelques espèces de la tribu des génistées (légumineuses). Can J Bot 65, 1870-1875

Citharel J, Delamarre D (1989) Synthesis of $\gamma$ conglutin during seed maturation in Lupinus albus. Plant Physiol Biochem 27, 211-218

Davey JE, Van Staden J (1978) Ultrastructural aspects of reserve protein deposition during cotyledonary cell development in Lupinus albus. $Z$ Pflanzenphysiol 89, 259-271

Deckert JE, Gwozdz EA (1991) Storage globulins synthesised in vitro by polyribosomes and mRNA from developing seeds of Lupinus luteus. Plant Physiol Biochem 29, 269-280

Duranti M, Faoro F, Harris N (1991) Immunocytochemical localization of conglutin $\gamma$ and legumin-like globulin in developing and mature seeds of Lupinus albus L. Protoplasma 161, 104-110

Esnault MA, Klingler J, Citharel J (1992) Étude de la fraction conglutine $\delta$ de la graine de lupin jaune. $I n$ : 1 er congr eur protéagineux, Angers (AEP, organisateur). Recueil de communications. Soft Publicité, Reims, 183-184

Esnault MA, Merceur A, Citharel J (1991) Characterization of yellow lupin seeds. Plant Physiol Biochem 29, 573-583

Gayler KR, Boadle BG, Snook M, Johnson ED (1984) Precursors of storage proteins in Lupinus angustifolius. Biochem J 221, 333-341

Gayler KR, Kolivas S, Macfarlane AJ, Lilley GG, Baldi M, Blagrove RJ, Johnson ED (1990) Biosynthesis, cDNA and amino acid sequences of a precursor of conglutin $\delta$, a sulphur-rich protein from Lupinus angustifolius. Plant Mol Biol 15, 879-893
Gayler KR, Wachsmann K, Kolivas S, Nott R, Johnson ED (1989) Isolation and characterization of protein bodies in Lupinus angustifolius. Plant Physiol 91, 1425-1431

Johnson ED, Knight J, Gayler KR (1985) Biosynthesis and processing of legumin-like storage proteins in $\mathrm{Lu}$ pinus angustifolius (lupin). Biochem J 232, 673-679

Joubert FJ (1955) Lupin seed proteins. II. A physicochemical study of the proteins from yellow lupin seed (Lupinus luteus). Biochim Biophys Acta 17, 444-445

Laemmli UK (1970) Cleavage of structural proteins during the assembly of the head of bacteriophage T4 Nature 227, 680-685

Le Gal MF, Rey L (1986) The reserve proteins in the cells of mature cotyledons of Lupinus albus var Lucky. I. Quantitative ultrastructural study of the protein bodies. Protoplasma 130, 120-127

Lilley GG (1986) Isolation of conglutin $\delta$, a sulphur-rich protein from the seeds of Lupinus angustifolius $\mathrm{L}$. $J$ Sci Food Agric 37, 1-11

Lilley GG, Inglis AS (1986) Amino acid sequence of conglutin $\delta$, a sulphur-rich seed protein of Lupinus angustifolius L. FEBS 195, 235-241

Lott JNA (1981) Protein bodies in seeds. Nord $J$ Bot 1 , 421-432

Lott JNA, Buttrose MS (1978) Globoids in protein bodies of legume seed cotyledons. Aust J Plant Physiol 5, 89-111

Mlodzianowski $F$ (1978) The fine structure of protein bodies in lupine cotyledons during the course of seed germination. $Z$ Pflanzenphysiol 86, 1-13

Restani P, Duranti M, Cerletti P, Simonetti P (1981) Subunit composition of the seed globulins of Lupinus albus. Phytochemistry 20, 2077-2083

Sato K, Yamagishi T, Kamata Y, Yamauchi F (1987) Subunit structure and immunological properties of a basic $7 \mathrm{~S}$ globulin from soybean seeds. Phytochemistry 26, 903-908

Sobolev AM, Buzulukova NP, Dimitrievam I, Barbashova AK (1976) Structural-biochemical organization of aleurone grains in seeds of yellow lupin. Soviet PI Physiol 23, 621-628

Wozny A, Mlodzianowski F, Stefaniak B (1984) Protein bodies formation in yellow lupin seeds. Acta Soc Bot Pol 53, 3-9 